\title{
Complementary colors of colorons: the elementary excitations of the SU(3) Haldane-Shastry model
}

\author{
Dirk Schuricht and Martin Greiter \\ Institut für Theorie der Kondensierten Materie, \\ Universität Karlsruhe, Postfach 6980, 76128 Karlsruhe, Germany
}

\begin{abstract}
We propose two possible trial wave functions for the elementary excitations of the SU(3) HaldaneShastry model, but then argue on very general grounds that only one or the other can be a valid excitation. We then prove explicitly that the trial wave function describing a coloron excitation which transforms according to representation $\overline{3}$ under $\mathrm{SU}(3)$ rotations if the spins of the original model transform according to representation 3, is exact. If a basis for the spins on the chain is spanned by the colors blue, red, and green, a basis for the coloron excitations is hence given by the complementary colors yellow, cyan, and magenta. We derive the dispersion and show that colorons exist only in one third of the Brillouin zone. We further obtain the exclusion statistics among polarized colorons and discuss the generalization to $\mathrm{SU}(n)$.

PACS numbers: 75.10.Pq, 02.30.Ik, 75.10.Jm
\end{abstract}


In recent years, there has been a substantial interest in models in condensed matter physics with symmetry groups larger than $\mathrm{SU}(2)$, the group underlying the usual spin algebra. In particular, models with SU(4) symmetry have been subject to detailed investigations [1-6], as such models can be realized experimentally in transition-metal oxides [7-9] where the electron spin is coupled to orbital degrees of freedom. Honerkamp and Hofstetter [10] have investigated the SU(n) generalization of the Hubbard model, proposing possible experimental realizations in ultracold fermion systems [11, 12].

In this Letter, we will investigate yet another model in this general class, the SU(3) Haldane-Shastry model (HSM) [13-20], which serves as a paradigm for not only the SU(3) spin chain, but, as we shall see, also illustrates some very general properties of $\mathrm{SU}(n)$ chains. In particular, we derive the quantum numbers of the elementary, fractionally quantized excitations, the analogs of the spinon excitations in $\mathrm{SU}(2)$, which we call colorons for $\mathrm{SU}(3)$. One of the key results is that these excitations transform under the $\mathrm{SU}(3)$ representation conjugate to the representation of the original SU(3) spins localized at the sites of the chain (see Fig. 1). First, this result reveals a new feature of fractional quantization, namely the occurence of the conjugate representation or complementary colors. This feature is meaningless in the case of $\mathrm{SU}(2)$ spin chains or the fractional quantum Hall effect, but significant in all other instances of fractional quantization in $\mathrm{SU}(n)$ chains and possible higher dimensional liquids, regardless of model specifics. Second, as a consequence of their complementary colors the coloron excitations exist only in one third of the Brillouin zone (BZ). This is reflected in the dynamical structure factor (DSF) calculated exactly by Yamamoto et al. [21, 22]. We hence provide an explicit interpretation of the DSF in terms of wave functions, which can be used as starting point for the investigation of other SU(3) spin chains as well. In our analysis, however, we will focus exclusively on the SU(3) HSM, as this is the simplest model in which this general result can be illustrated through exact solution, and thereby demonstrate the amenability of the SU(3) HSM to exact solution in terms of explicit wave functions.

The SU(3) $1 / r^{2}$ or Haldane-Shastry model $[17,19]$ is most conveniently formulated by embedding the one-dimensional chain with periodic boundary conditions into the complex plane by mapping it onto the unit circle with the $\mathrm{SU}(3)$ spins located at complex positions $\eta_{\alpha}=\exp \left(i \frac{2 \pi}{N} \alpha\right)$, where $N$ denotes the number of sites and $\alpha=1, \ldots, N$. The Hamiltonian 
is given by

$$
H_{\mathrm{SU}(3)}=\left(\frac{2 \pi}{N}\right)^{2} \sum_{\alpha<\beta}^{N} \frac{\boldsymbol{J}_{\alpha} \cdot \boldsymbol{J}_{\beta}}{\left|\eta_{\alpha}-\eta_{\beta}\right|^{2}},
$$

where $\boldsymbol{J}_{\alpha}=\frac{1}{2} \sum_{\sigma \tau} c_{\alpha \sigma}^{\dagger} \boldsymbol{\lambda}_{\sigma \tau} c_{\alpha \tau}$ is the 8-dimensional SU(3) spin vector, $\boldsymbol{\lambda}$ a vector consisting of the 8 Gell-Mann matrices [23], and $\sigma$ and $\tau$ are $\mathrm{SU}(3)$ spin or color indices, which take the values blue (b), red (r), or green (g) (see Fig. 1). For all practical purposes, it is convenient to express $H_{\mathrm{SU}(3)}$ directly in terms of colorflip operators $e_{\alpha}^{\sigma \tau} \equiv c_{\alpha \sigma}^{\dagger} c_{\alpha \tau}$ :

$$
H_{\mathrm{SU}(3)}=\frac{2 \pi^{2}}{N^{2}} \sum_{\alpha<\beta}^{N} \sum_{\sigma \tau} \frac{e_{\alpha}^{\sigma \tau} e_{\beta}^{\tau \sigma}-\frac{1}{27}}{\left|\eta_{\alpha}-\eta_{\beta}\right|^{2}}
$$

where the color sum includes terms with $\sigma=\tau$. The ground state of $H_{\mathrm{SU}(3)}$ for $N=3 M$ ( $M$ integer) is most easily formulated by Gutzwiller projection of a filled band (or Slater determinant (SD) state) containing a total of $N$ SU(3) particles obeying Fermi statistics (see Fig. 2a)

$$
\left|\Psi_{0}\right\rangle=P_{\mathrm{G}} \prod_{|q| \leq q_{\mathrm{F}}} c_{q \mathrm{~b}}^{\dagger} c_{q \mathrm{r}}^{\dagger} c_{q \mathrm{~g}}^{\dagger}|0\rangle \equiv P_{\mathrm{G}}\left|\Psi_{\mathrm{SD}}^{N}\right\rangle .
$$

The Gutzwiller projector $P_{\mathrm{G}}$ eliminates configurations with more than one particle on any site, and, as the total number of particles equals the total number of sites, thereby effectively enforces single occupancy on all sites. As $\left|\Psi_{\mathrm{SD}}^{N}\right\rangle$ is an $\mathrm{SU}(3)$ singlet by construction and $P_{\mathrm{G}}$ commutes with $\mathrm{SU}(3)$ rotations, $\left|\Psi_{0}\right\rangle$ is an $\mathrm{SU}(3)$ singlet as well. The model is fully integrable even for a finite number of sites; the algebra of the (infinite) number of conserved quantities is generated by the $\mathrm{SU}(3)$ total spin and rapidity operators

$$
\boldsymbol{J}=\sum_{\alpha=1}^{N} \boldsymbol{J}_{\alpha}, \quad \Lambda^{a}=\frac{1}{2} \sum_{\alpha \neq \beta}^{N} \frac{\eta_{\alpha}+\eta_{\beta}}{\eta_{\alpha}-\eta_{\beta}} f^{a b c} J_{\alpha}^{b} J_{\beta}^{c},
$$

which commute with the Hamiltonian but do not commute mutually $\left(a=1, \ldots, 8\right.$ and $f^{a b c}$ are the structure constants of $\mathrm{SU}(3)$ defined through $\left.\left[\lambda^{a}, \lambda^{b}\right]=2 f^{a b c} \lambda^{c}\right)$.

If one interprets the state $\left|0_{\mathrm{g}}\right\rangle \equiv \prod_{\alpha=1}^{N} c_{\alpha \mathrm{g}}|0\rangle$ as a reference state and the colorflip operators $e^{\mathrm{bg}}$ and $e^{\mathrm{rg}}$ as "particle creation operators", the ground state $(3)$ can be rewritten as $[18,19]$

$$
\left|\Psi_{0}\right\rangle=\sum_{\left\{z_{i}, w_{k}\right\}} \Psi_{0}\left[z_{i} ; w_{k}\right] e_{z_{1}}^{\mathrm{bg}} \ldots e_{z_{M}}^{\mathrm{bg}} e_{w_{1}}^{\mathrm{rg}} \ldots e_{w_{M}}^{\mathrm{rg}}\left|0_{\mathrm{g}}\right\rangle,
$$

where the sum extends over all possible ways to distribute the positions of the blue particles $z_{1}, \ldots, z_{M}$ and red particles $w_{1}, \ldots, w_{M}$ over the $N$ sites. The ground state wave function 


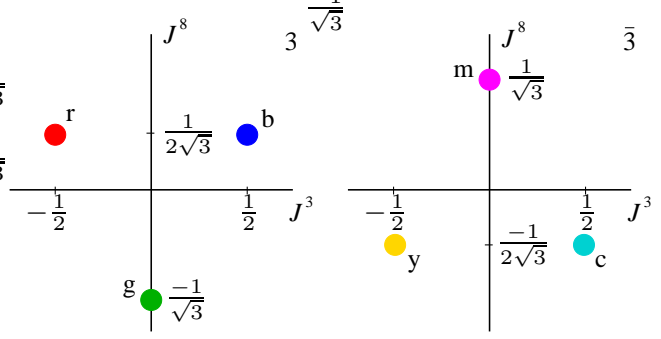

FIG. 1: Weight diagrams of the $\mathrm{SU}(3)$ representations 3 and $\overline{3} . J^{3}$ and $J^{8}$ denote the diagonal generators [23].

is given by (see Fig. 2b)

$$
\Psi_{0}\left[z_{i} ; w_{k}\right] \equiv \prod_{i<j}^{M_{1}}\left(z_{i}-z_{j}\right)^{2} \prod_{k<l}^{M_{2}}\left(w_{k}-w_{l}\right)^{2} \prod_{i=1}^{M_{1}} \prod_{k=1}^{M_{2}}\left(z_{i}-w_{k}\right) \prod_{i=1}^{M_{1}} z_{i} \prod_{k=1}^{M_{2}} w_{k}
$$

with $M_{1}=M_{2}=M$. The ground state energy is

$$
E_{0}=-\frac{\pi^{2}}{18}\left(N+\frac{7}{N}\right)
$$

The total momentum, as defined through $e^{i p}=\Psi_{0}\left[\eta_{1} z_{i}, \eta_{1} w_{k}\right] / \Psi_{0}\left[z_{i}, w_{k}\right]$ with $\eta_{1}=\exp \left(i \frac{2 \pi}{N}\right)$, is $p=0$ regardless of $M$ (not true for $\mathrm{SU}(2)$ ).

We now turn to the heart of our analysis, the elementary and fractionally quantized excitations, which we call colorons. In principle, there are two possible, non-equivalent constructions for localized excitations starting from (3). We may either create a particle with color $\sigma$ on a chain with $N=3 M+1$ sites before Gutzwiller projection,

$$
\left|\Psi_{\gamma \sigma}^{\mathrm{c}}\right\rangle=P_{\mathrm{G}} c_{\gamma \sigma}^{\dagger}\left|\Psi_{\mathrm{SD}}^{N-1}\right\rangle
$$

or annihilate a particle with color $\sigma$ on a chain with $N=3 M-1$ :

$$
\left|\Psi_{\gamma \bar{\sigma}}^{\mathrm{a}}\right\rangle=P_{\mathrm{G}} c_{\gamma \sigma}\left|\Psi_{\mathrm{SD}}^{N+1}\right\rangle
$$
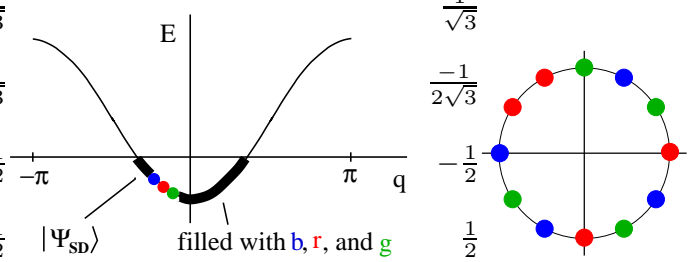

FIG. 2: a) Total antisymmetric $N$-particle state. b) Typical configuration in $\left|\Psi_{0}\right\rangle$. 
In both cases, $c_{\gamma \sigma}^{\dagger}$ or $c_{\gamma \sigma}$ creates an inhomogeneity in color and charge before projection. The projection once again enforces one particle per site and thereby removes the charge inhomogeneity, while it commutes with color and thereby preserves the color inhomogeneity. Consequently, the trial states $\left|\Psi_{\gamma \sigma}^{\mathrm{c}}\right\rangle$ and $\left|\Psi_{\gamma \bar{\sigma}}^{\mathrm{a}}\right\rangle$ describe localized "excitations" of color $\sigma$ or complementary color $\bar{\sigma}$, respectively, at lattice site $\eta_{\gamma}$. Of course, since $H_{\mathrm{SU}(3)}$ is translationally invariant, we expect neither of them, but only momentum eigenstates constructed from them via

$$
\left|\Psi_{n}\right\rangle \equiv \sum_{\gamma=1}^{N} e^{-i \frac{2 \pi}{N} \gamma n}\left|\Psi_{\gamma}\right\rangle=\sum_{\gamma=1}^{N}\left(\bar{\eta}_{\gamma}\right)^{n}\left|\Psi_{\gamma}\right\rangle,
$$

where $n$ is a momentum quantum number, to be eigenstates.

The important thing to realize now is that only (8) or (9), but not both, can describe a valid excitation. To see this, let us assume $\sigma=\mathrm{b}$ for ease in presentation, and note that (8) is apart from a normalization factor equivalent to

$$
\left|\Psi_{\gamma \mathrm{b}}^{\mathrm{c}}\right\rangle=P_{\mathrm{G}} c_{\gamma \mathrm{r}} c_{\gamma \mathrm{g}}\left|\Psi_{\mathrm{SD}}^{N+2}\right\rangle
$$

i.e., creation of a blue particle before projection is tantamount to annihilation of both a red and a green particle at site $\eta_{\gamma}$. If momentum eigenstates constructed from $\left|\Psi_{\gamma \bar{\sigma}}^{\mathrm{a}}\right\rangle$ via (10) were energy eigenstates, the anti-red (cyan) and anti-green (magenta) coloron excitations in (11) would individually seek to be momentum eigenstates, which implies that a trial wave function forcing them to sit on the same site would not yield an energy eigenstate. The same argument can be made the other way round.

In the following, we show by explicit calculation that momentum eigenstates constructed from $\left|\Psi_{\gamma \bar{\sigma}}^{\mathrm{a}}\right\rangle$ via (10) are exact eigenstates of $H_{\mathrm{SU}(3)}$ with momentum

$$
p=\frac{4}{3} \pi-\frac{2 \pi}{N}\left(n+\frac{1}{3}\right), \quad 0 \leq n \leq \frac{N-2}{3},
$$

(where $n$ is shifted with respect to $n$ in (10) by a constant depending on which momenta are occupied in the Slater determinant state) and energy

$$
E(p)=E_{0}+\frac{2}{9} \frac{\pi^{2}}{N^{2}}+\frac{3}{4}\left(\frac{\pi^{2}}{9}-(p-\pi)^{2}\right)
$$

(see Fig. 3). For $(N-2) / 3<n<N,\left|\Psi_{n \bar{\sigma}}^{\mathrm{a}}\right\rangle$ vanishes identically. The color $\bar{\sigma}$ of the excitation, constructed by annihilation of a particle of color $\sigma$ from an overall color singlet $\left|\Psi_{\mathrm{SD}}^{N+1}\right\rangle$, hence transforms according to the representation $\overline{3}$ conjugate to the fundamental 


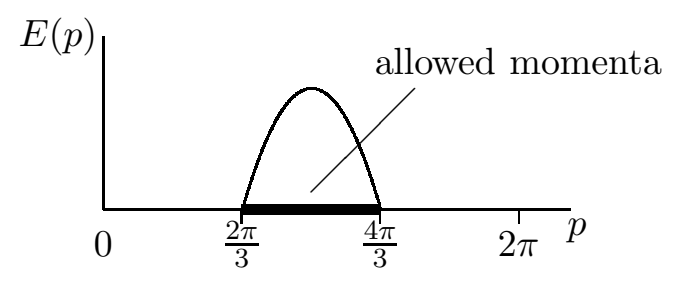

FIG. 3: One-coloron dispersion relation.

representation 3 of the original particles on the sites of the chain. This is consistent with results on the spectrum of the $\mathrm{SU}(3)_{1}$ Wess-Zumino-Witten (WZW) model obtained by Bouwknegt and Schoutens $[24,25]$. The momenta of the coloron excitations $\left|\Psi_{n \bar{\sigma}}\right\rangle$ fill only one third of the BZ, which is fully consistent with the calculations of the DSF [21, 22].

For simplicity, we choose $\bar{\sigma}=\overline{\mathrm{b}}=\mathrm{y}$ (an anti-blue or yellow coloron) and express $\left|\Psi_{\gamma \overline{\mathrm{b}}}^{\mathrm{a}}\right\rangle$ through the corresponding wave function

$$
\Psi_{\gamma}\left[z_{i} ; w_{k}\right]=\prod_{i=1}^{M_{1}}\left(\eta_{\gamma}-z_{i}\right) \Psi_{0}\left[z_{i} ; w_{k}\right] \equiv \psi_{\gamma} \Psi_{0} .
$$

with $\Psi_{0}$ given by $(6)$ and $M_{1}=(N-2) / 3, M_{2}=(N+1) / 3$. The momentum eigenstate $\left|\Psi_{n \bar{b}}^{\mathrm{a}}\right\rangle$ is then given by

$$
\Psi_{n}\left[z_{i} ; w_{k}\right]=\sum_{\gamma=1}^{N}\left(\bar{\eta}_{\gamma}\right)^{n} \Psi_{\gamma}\left[z_{i} ; w_{k}\right]
$$

which vanishes identically unless $0 \leq n \leq M_{1}$. To evaluate the action of $H_{\mathrm{SU}(3)}$ on (15) we first replace $e_{\alpha}^{\mathrm{gg} g} e_{\beta}^{\mathrm{gg}}$ in (2) by $\left(1-e_{\alpha}^{\mathrm{bb}}-e_{\alpha}^{\mathrm{rr}}\right)\left(1-e_{\beta}^{\mathrm{bb}}-e_{\beta}^{\mathrm{rr}}\right)$ and treat each term separately [26]. For example, the term $\left[e_{\alpha}^{\mathrm{bg}} e_{\beta}^{\mathrm{gb}} \Psi_{\gamma}\right]\left[z_{i} ; w_{k}\right]$, which vanishes unless one of the $z_{i}$ 's is equal to $\eta_{\alpha}$, yields through Taylor expansion

$$
\begin{aligned}
{\left[\sum_{\alpha \neq \beta}^{N} \frac{e_{\alpha}^{\mathrm{bg}} e_{\beta}^{\mathrm{gb}}}{\left|\eta_{\alpha}-\eta_{\beta}\right|^{2}} \Psi_{\gamma}\right]\left[z_{i} ; w_{k}\right]=} & \sum_{i=1}^{M_{1}} \sum_{m=0}^{N-1} \frac{A_{m} z_{i}^{m+1}}{m !} \frac{\partial^{m}}{\partial z_{i}^{m}} \frac{\Psi_{\gamma}}{z_{i}} \\
= & -\frac{N^{3}+12 N^{2}-123 N+190}{324} \Psi_{\gamma} \\
& -\frac{N-3}{2} \sum_{i=1}^{M_{1}} \sum_{k=1}^{M_{2}} \frac{z_{i}}{z_{i}-w_{k}} \Psi_{\gamma}+\sum_{i \neq j}^{M_{1}} \frac{z_{i}^{2}}{\left(z_{i}-z_{j}\right)^{2}} \Psi_{\gamma} \\
& +2 \sum_{i \neq j}^{M_{1}} \sum_{k=1}^{M_{2}} \frac{z_{i}^{2}}{\left(z_{i}-z_{j}\right)\left(z_{i}-w_{k}\right)} \Psi_{\gamma} \\
& +\frac{1}{2} \sum_{i=1}^{M_{1}} \sum_{k \neq l}^{M_{2}} \frac{z_{i}^{2}}{\left(z_{i}-w_{k}\right)\left(z_{i}-w_{l}\right)} \Psi_{\gamma}
\end{aligned}
$$




$$
\begin{aligned}
& +\Psi_{0} \sum_{i=1}^{M_{1}}\left(\frac{z_{i}^{2}}{2} \partial_{i}^{2}+\sum_{j \neq i}^{M_{1}} \frac{2 z_{i}^{2}}{z_{i}-z_{j}} \partial_{i}-\frac{N-3}{2} z_{i} \partial_{i}\right) \psi_{\gamma} \\
& +\Psi_{0} \sum_{i=1}^{M_{1}} \sum_{k=1}^{M_{2}} \frac{z_{i}^{2}}{z_{i}-w_{k}} \partial_{i} \psi_{\gamma}
\end{aligned}
$$

where we have used $\operatorname{deg}_{z_{i}} \Psi_{\gamma}\left[z_{i} ; w_{k}\right]=N-1$ and defined $\partial_{i} \equiv \partial / \partial z_{i}, A_{m} \equiv-\sum_{\alpha=1}^{N-1} \eta_{\alpha}^{2}\left(\eta_{\alpha}-\right.$ $1)^{m-2}$. Evaluation of the latter yields $A_{0}=(N-1)(N-5) / 12, A_{1}=-(N-3) / 2, A_{2}=1$, and $A_{m}=0$ for $2<m \leq N-1$ [27]. The action of $\sum e_{\alpha}^{\mathrm{bg}} e_{\beta}^{\mathrm{gb}} /\left|\eta_{\alpha}-\eta_{\beta}\right|^{2}$ on $\Psi_{n}$ is obtained by Fourier transformation of (16); the first six terms on the RHS can be treated in analogy to the SU(3) HS ground state [19] and the calculations in the $\mathrm{SU}(2) \mathrm{HS}$ model [13, 27].

To mention another term,

$$
\begin{aligned}
{\left[\sum_{\alpha \neq \beta}^{N} \frac{e_{\alpha}^{\mathrm{br}} e_{\beta}^{\mathrm{rb}}}{\left|\eta_{\alpha}-\eta_{\beta}\right|^{2}} \Psi_{n}\right]\left[z_{i} ; w_{k}\right]=} & \frac{1}{N} \sum_{\gamma=1}^{N}\left(\bar{\eta}_{\gamma}\right)^{n} \sum_{i=1}^{M_{1}} \sum_{k=1}^{M_{2}} \frac{z_{i} w_{k}}{\left(z_{i}-w_{k}\right)^{2}}\left(1+\frac{z_{i}-w_{k}}{\eta_{\gamma}-z_{i}}\right) \\
& \cdot \prod_{j \neq i}^{M_{1}} \frac{z_{j}-w_{k}}{z_{j}-z_{i}} \prod_{l \neq k}^{M_{2}} \frac{w_{l}-z_{i}}{w_{l}-w_{k}} \Psi_{\gamma}\left[z_{i} ; w_{k}\right],
\end{aligned}
$$

splits into two parts: one, which can again be treated as for the ground state, and another, the sum of which with the last term in (16) can be simplified using

$$
\begin{aligned}
\sum_{i=1}^{M_{1}} \sum_{k=1}^{M_{2}} \frac{z_{i}}{z_{i}-w_{k}} \cdot\left[w_{k} \prod_{j \neq i}^{M_{1}} \frac{z_{j}-w_{k}}{z_{j}-z_{i}} \prod_{l \neq k}^{M_{2}} \frac{w_{l}-z_{i}}{w_{l}-w_{k}}-z_{i}\right] \sum_{\gamma=1}^{N}\left(\bar{\eta}_{\gamma}\right)^{n} \prod_{j \neq i}^{M_{1}}\left(\eta_{\gamma}-z_{j}\right) \\
=\left[-\frac{1}{2} n(n+1)+\frac{1}{2} M_{1}\left(M_{1}+1\right)\right] \sum_{\gamma=1}^{N}\left(\bar{\eta}_{\gamma}\right)^{n} \prod_{i=1}^{M_{1}}\left(\eta_{\gamma}-z_{i}\right),
\end{aligned}
$$

which is valid for $M_{1} \leq M_{2}$ [26]. In this manner, the action of every term in (2) on (15) can be evaluated, yielding that (15) is indeed an eigenstate with energy (13) and momentum (12).

It is straightforward to read off the quantum or exclusion statistics [28] of color-polarized colorons. Consider a chain with $N=3 M-1$ sites and a single yellow coloron. According to (3), (9), and (10), there are as many single particle orbitals available to the coloron as there are blue particles in the Slater determinant state, that is, $M$. If we now were to create three additional yellow colorons, the Slater determinant state would have to contain three more particles, one of each color. This implies there would be one additional orbital, while the three additional colorons would occupy three orbitals, meaning that the number of orbitals available for our original coloron would be reduced by two. The statistical parameter is 
hence given by $g=2 / 3$. The fractional statistics manifests itself further in the exponents of the algebraic decay of the form factor of the DSF [21, 22], in the thermodynamics of the model [29, 30], as well as the S-matrix evaluated with the asymptotic Bethe Ansatz [31]. A similar exclusion statistics exists in the conformal field theory spectrum of WZW models $[25$, 32]. The general exclusion statistics and state counting for $\mathrm{SU}(n)(n \geq 3)$ spin chains is highly non-trivial and will be subject of a future publication [33].

The results derived here generalize directly to the SU(n) HSM [26]. The eigenstates describing a single fractionally quantized $\mathrm{SU}(n)$ spinon excitation are given by the $\mathrm{SU}(n)$ generalization of (9). If the particles on the sites of the chain transform under the fundamental representation $n$ of $\mathrm{SU}(n)$, the spinons transform under the conjugate representation $\bar{n}$. The allowed momenta of the spinons are restricted to $p \in\left[\pi-\frac{\pi}{n}, \pi+\frac{\pi}{n}\right]$ (or $\left[-\frac{\pi}{n}, \frac{\pi}{n}\right]$ if $n$ even and $(N+1) / n$ odd), and hence fill only the $n^{\text {th }}$ part of the BZ, with dispersion

$$
E^{n}(p)=E_{0}^{n}+\frac{n^{2}-1}{12 n} \frac{\pi^{2}}{N^{2}}+\frac{n}{4}\left(\frac{\pi^{2}}{n^{2}}-(p-\pi)^{2}\right),
$$

where $E_{0}^{n}$ is the ground state energy $[18,19]$. The statistical parameter for polarized spinons is given by $(n-1) / n$.

In conclusion, we have used the HSM to make the case that the elementary excitations of $\mathrm{SU}(n)$, but in particular $\mathrm{SU}(3)$, spin chains transform under the representation conjugate to the representation of the $\mathrm{SU}(n)$ spins on the chain, exist only in one $n^{\text {th }}$ of the Brillouin zone, and obey fractional statistics with $g=(n-1) / n$.

One of us (DS) was supported by the German Research Foundation (DFG) through GK 284. The other (MG) would like to thank the organizers of the 2003 Amsterdam Summer Workshop on Flux, Charge, Topology and Statistics, where this work was partially inspired.

[1] Y. Q. Li et al., Phys. Rev. Lett. 81, 3527 (1998).

[2] B. Frischmuth et al., Phys. Rev. Lett. 82, 835 (1999).

[3] P. Azaria et al., Phys. Rev. Lett. 83, 624 (1999).

[4] Y. Q. Li et al., Phys. Rev. B 60, 12781 (1999).

[5] C. Itoi et al., Phys. Rev. B 61, 6747 (2000).

[6] S. J. Gu and Y. Q. Li, Phys. Rev. B 66, 092404 (2002).

[7] Y. Tokura and N. Nagaosa, Science 288, 462 (2000). 
[8] M. Isobe and Y. Ueda, J. Phys. Soc. Jpn. 65, 1178 (1996).

[9] Y. Fujii et al., J. Phys. Soc. Jpn. 66, 326 (1997).

[10] C. Honerkamp and W. Hofstetter, Phys. Rev. Lett. 92, 170403 (2004).

[11] E. R. I. Abraham et al., Phys. Rev. A 55, R3299 (1997).

[12] C. A. Regal and D. S. Jin, Phys. Rev. Lett. 90, 230404 (2003).

[13] F. D. M. Haldane, Phys. Rev. Lett. 60, 635 (1988).

[14] B. S. Shastry, Phys. Rev. Lett. 60, 639 (1988).

[15] F. D. M. Haldane, Phys. Rev. Lett. 66, 1529 (1991).

[16] F. D. M. Haldane et al., Phys. Rev. Lett. 69, 2021 (1992).

[17] N. Kawakami, Phys. Rev. B 46, 1005 (1992).

[18] N. Kawakami, Phys. Rev. B 46, R3191 (1992).

[19] Z. N. C. Ha and F. D. M. Haldane, Phys. Rev. B 46, 9359 (1992).

[20] Z. N. C. Ha and F. D. M. Haldane, Phys. Rev. B 47, 12459 (1993).

[21] T. Yamamoto et al., Phys. Rev. Lett. 84, 1308 (2000).

[22] T. Yamamoto et al., J. Phys. Soc. Jpn. 69, 900 (2000).

[23] H. Georgi, Lie Algebras in Particle Physics (Addison-Wesley, Redwood City, 1982).

[24] P. Bouwknegt and K. Schoutens, Nucl. Phys. B482, 345 (1996).

[25] K. Schoutens, Phys. Rev. Lett. 79, 2608 (1997).

[26] D. Schuricht and M. Greiter, manuscript in preparation.

[27] B. A. Bernevig et al., Phys. Rev. B 64, 24425 (2001).

[28] F. D. M. Haldane, Phys. Rev. Lett. 67, 937 (1991).

[29] Y. Kuramoto and Y. Kato, J. Phys. Soc. Jpn. 64, 4518 (1995).

[30] Y. Kato and Y. Kuramoto, J. Phys. Soc. Jpn. 65, 1622 (1996).

[31] F. H. L. Eßler, Phys. Rev. B 51, 13357 (1995).

[32] P. Bouwknegt and K. Schoutens, Nucl. Phys. B547, 501 (1999).

[33] M. Greiter and D. Schuricht, manuscript in preparation. 